\title{
Twenty years of RNA: reflections on post-transcriptional regulation
}

\author{
WILLIAM "BILL" F. MARZLUFF \\ Department of Biochemistry and Biophysics, Integrative Program for Biological and Genome Sciences, University of North Carolina, \\ Chapel Hill, North Carolina 27599, USA
}

In retrospect, the establishment of the RNA journal 20 years ago came at a propitious time in the study of RNA biology. In the previous decade the discovery of catalytic RNA, and the variety of different RNA processing pathways required for synthesis of mature RNAs, had expanded our view of RNA from simply being a passive template for carrying the information in DNA for protein sequences to the ribosome for translation, to a much broader role in the cell. The concept of the RNA world had been embraced (at least by RNA biologists). The broad picture of much of RNA metabolism had been elucidated but the details of the complexities of gene expression implied by alternative splicing, alternative polyadenylation and the details of RNA metabolism, including RNA transport and RNA degradation still needed to be worked out. The decade of new discoveries prior to establishment of the RNA journal clearly showed the broad spectrum of problems remaining to be solved in both the structure and function of RNA.

What was not expected, at least to me, was the broad range of different RNA molecules with diverse functions that have been discovered in the last 20 years (and the feeling that there are still many more that we can look forward to discovering in the future). The RNA journal has played a key role in bringing a single portal which discoveries in the broad range of RNA biology, from fundamental studies of structure and dynamics of RNA molecules and RNA-protein complexes, to the diverse roles of RNA in development, are collected bringing them to the immediate attention of everyone working in RNA. The rapidity with which the RNA journal achieved the stature of a major journal is impressive. Within its first decade, if not sooner, it had achieved recognition as the place to find advances in all areas of RNA biology. Looking at publications from my group, which have been focused on different aspects of RNA for almost 40 years, we have published more papers in $R N A$ in the last decade than in any other journal. Clearly this has become the journal of choice for us; it is a

\footnotetext{
Corresponding author: william_marzluff@med.unc.edu

Article and publication date are at http://www.rnajournal.org/cgi/doi/ 10.1261/rna.050997.115. Freely available online through the RNA Open Access option.
}

place where we know the reviewers are knowledgable and competent, and especially where the editors are willing to adjudicate decisions among differing reviews. The breadth of the topics covered in the journal are impressive and we know that our own work will reach a broad audience, whether it is a study of a new RNA-protein complex or a study of the genetics of an RNA processing factor.

The development of RNA-based technologies has been one impressive field, with the initial discovery of RNA interference resulting in the development of siRNA approaches for probing cellular regulation by scientists in all fields. I teach a first-year graduate course focused on post-transcriptional regulation and each year try to select a paper published since the previous course, which introduces a new method or approach which seems likely to change or expand the approaches available to solve biological problems. Since often when one reads or hears a talk about a new technique, the hoped-for promise of the new findings never really pan out, I tell the students to be cautious about trying every new thing they hear about. More often than not over the last 15 years, I find that a "game-changing" advance, either a technical or conceptual clear scientific breakthrough involving RNA has been made. A striking example was the string of discoveries which resulted from the discovery of RNA interference. This pathway initially seemed possibly to be limited in application to a subset of species. The discovery that using small siRNAs was effective in knocking down gene expression in mammalian cells by Tuschl and coworkers in 2001, opened up a new approach to study of the role of proteins in mammalian cells, resulting in a huge demand for synthetic RNA molecules, and many advances in all areas of biology. The next year, the finding that miRNAs, originally described with two examples over the course of a decade in C. elegans, were found in all metazoans, opened up a completely new field of regulation by RNA. Within a few years the general mechanism of miRNA biosynthesis and the mechanism of cleavage by Ago2 had been elucidated, followed by many

(C) 2015 Marzluff This article, published in $R N A$, is available under a Creative Commons License (Attribution-NonCommercial 4.0 International), as described at http://creativecommons.org/licenses/by-nc/4.0/. 
examples of miRNA roles in biology, including the recognition of an important role in embryonic development, as well as in the development of some cancers. Over the next few years the mechanism of miRNA action on gene expression was studied in many groups, including a memorable session at the RNA Meeting in Seattle where talks from several groups each supporting a different mechanism of miRNA action using essentially the same reporter genes were presented. Discussing the complexities of these studies, and the evidence leading to the different conclusions on how miRNAs regulate gene expression with the classes over the next few years, gave the students some appreciation for the difficulty of separating the different possible molecular steps in gene expression. While this question has now been largely resolved with the recognition that the RISC complex can have multiple separate effects on translation and mRNA stability, I can't recall another area of such intense investigation by a large number of laboratories that resulted in so many different mechanisms being proposed, before some general consensus about the biochemical basis of miRNA action was finally reached (at least I think it has been reached).

New breakthroughs continue to occur every year: methods to identify the RNAs that a protein is bound to inside the cell; to determine the complexity of alternative polyadenylation, and its biological role; ribosome profiling to determine the relative rates of translation of mRNAs; the surprising finding that many "long non-coding RNAs" are actually translated and the relatively small polypeptides they produce are biologically functional. Strikingly within the last two years, another RNA based method for manipulating genes and gene expression, CrispR technology, which clearly has (or will) transform our ability to design experiments to understand gene function even more powerful than the siRNA technology. Although the advances I teach about are generally related to the biological functions of RNA, there have been equally impressive developments in understanding RNA structure, particularly the ribosome but also other large complex RNAs and RNPs.

Our own work has been focused on the regulation of a unique set of mRNAs, the metazoan replication-dependent histone mRNAs. Since these mRNAs are still the only known cellular eukaryotic mRNAs that are not polyadenylated, their metabolism must differ from the polyadenylated mRNAs. The discovery that the bulk of the regulation of these mRNAs to was post-transcriptional led to our having to investigate in detail histone mRNA metabolism and its regulation. Coincidently, our major advances in starting to understand the detailed mechanisms of regulation of these mRNAs, corresponded to the founding of the $R N A$ journal. We knew that mRNA degradation was regulated, but the biochemical mechanisms of mRNA degradation were entirely unknown. We also knew the cis element required for regulation, the stem-loop at the $3^{\prime}$ end of the mRNA, but not the factor that bound it. It took development of a new technology by Marv Wickens and Stan Fields to solve this problem. They developed the 3-hybrid system, designed to use an approach similar to the 2-hybrid system to identify proteins interacting with specific RNA sequences. I remember Marv presenting this system in the last talk on the first night of a Cold Spring Harbor meeting (as the bar was closing), where he decided not to talk about his submitted abstract. The small audience remaining immediately realized the potential of the system. To me this system seemed ideal for identifying our elusive factor. Indeed within two months after receiving the necessary reagents from Marv, Dani Schumperli's laboratory and my laboratory had cloned the factor, and we each presented the findings at the next RNA Society meeting. It turned out that the 3-hybrid system was wonderfully designed for isolating the stem-loop binding protein (SLBP), which bound a small defined structure with very high affinity, but for reasons that are still not clear was not used successfully to identify many other proteins.

The isolation of SLBP led to extensive work on post-transcriptional regulation of histone mRNA metabolism including details of the mechanism of histone pre-mRNA processing and the cell-cycle regulation of histone mRNA accomplished partly by the post-transcriptional regulation of SLBP. The finding that the endonuclease that processes histone mRNA is CPSF73, the same endonuclease that cleaves pre-mRNA leading to polyadenylation, was the first indication that there was significant overlap between the factors required for histone mRNA processing and cleavage/polyadenylation. The ultimate elucidation of the structure of the SLBP-RNA complex by Liang Tong a couple years ago, gave us the first real picture of part of this critical molecule that participates in all aspects of histone mRNA metabolism.

The second major advance necessary for our studies on post-transcriptional regulation was the determination of the pathways and factors involved in mRNA degradation led primarily by the laboratory of Roy Parker, using both yeast genetics and biochemical studies. At about the same time, an unusual mRNA decay pathway, nonsense-mediated decay (NMD) had been described in mammalian cells by Lynne Maquat, and the factors required for NMD were elucidated by Lynne's group and other groups. An intriguing feature of this pathway was that translation was absolutely required for mRNA degradation, a property that was also true for degradation of histone mRNAs. Taking advantage of the advances in our understanding of the basic mechanism of mRNA degradation, and the ability to manipulate the levels of different factors using siRNA technology, we published a series of papers on histone mRNA degradation, 18 years after our initial publications. Without the discoveries on the fundamental mechanisms of mRNA degradation, and the development of the siRNA technology, to allow us to manipulate the levels of different factors in mammalian cells, these studies would not have been possible.

The sequencing of genomes of the major organisms used in biomedical research in the early years of this century opened up a whole new dimension in our ability to 
understand all the RNAs expressed in an organism. However the more recent development of "next-generation" sequencing has truly revolutionized both the amount and types of experiments one can do. Sequencing DNA of a large number of organisms, as well as an increasing number of individuals, has provided a vast amount of information on the structure and evolution of genomes and genes. Moreover, for the RNA biologist it has opened up a vast array of novel experiments, and is only limited by the ability of the investigator to design novel strategies (and the funds to pay for the experiments), and the computational expertise to analyze the data obtained. No longer is it necessary to sequence hundreds or thousands of individual cDNA copies of RNA molecules to define the different microRNAs in a cell or alternatively spliced forms of mRNAs. All of the molecules present in a single sample could be identified and quantified in a single experiment. The ability to analyze in depth alternative splicing and alternative polyadenylation has resulted in a much more complete understanding of the transcriptome, and its regulation. The applications of this technology to RNA biology are just beginning to be exploited. Sequencing strategies to probe the structure of RNAs in cells have been developed and are starting to be applied. Our modest contribution has been the development of initial methods to probe histone mRNA degradation intermediates, allowing us for the first time to observe a wide variety of degradation intermediates that accumulate and deduce the major pathway of histone mRNA degradation. We had accumulated 50-100 degradation intermediates over about a four-year period (to the dismay of the postdoctoral fellows working on the project), from which it was impossible to deduce anything definitive, before visualizing thousands in a single experiment.

I look forward to the next decade (hopefully longer) of reading about, listening to and hopefully participating in, advances in RNA biology. Despite the basic nature of this area of research, the applications of the advances in RNA technology are now already in the clinic in trials to correct splicing defects in genetic diseases, as well as using RNA aptamers designed for specific applications in more common diseases. There is great hope that the CrispR technology may provide a mechanism, based on RNA biology, for true correction of genetic diseases. 

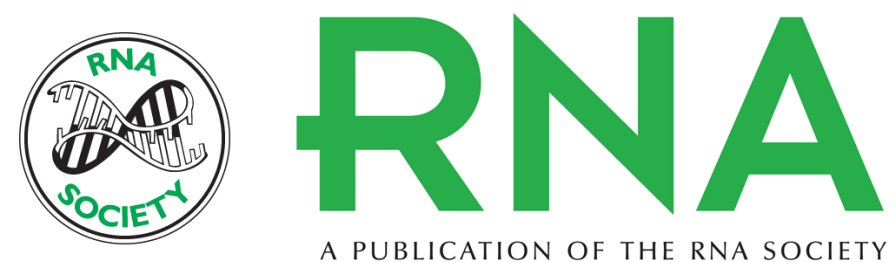

A PUBLICATION OF THE RNA SOCIETY

\title{
Twenty years of RNA: reflections on post-transcriptional regulation
}

\author{
William "Bill" F. Marzluff
}

RNA 2015 21: 687-689

\begin{aligned} & \hline Open Access Freely available online through the RNA Open Access option. \\ & $\begin{array}{r}\text { Creative } \\ \text { Commons } \\ \text { License }\end{array} \begin{array}{c}\text { This article, published in } R N A \text {, is available under a Creative Commons License } \\ \text { (Attribution-NonCommercial } 4.0 \text { International), as described at } \\ \text { http://creativecommons.org/licenses/by-nc/4.0/. }\end{array} \\ & \begin{aligned} \text { Email Alerting } \\ \text { Service }\end{aligned} \begin{array}{c}\text { Receive free email alerts when new articles cite this article - sign up in the box at the } \\ \text { top right corner of the article or click here. }\end{array} \\ &$\hline\end{aligned}

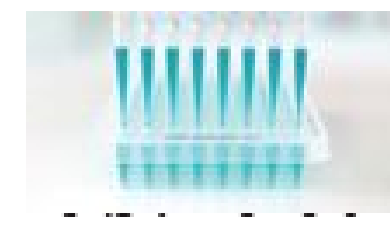

\section{Providing Precise Solutions for} your research.

To subscribe to RNA go to:

http://rnajournal.cshlp.org/subscriptions

(C) 2015 Marzluff; Published by Cold Spring Harbor Laboratory Press for the RNA Society 